\title{
Rolf H. Bremmer, Jr.: An Introduction to Old Frisian. History, Grammar, Reader, Glossary
}

\author{
Marti, Mirjam
}

DOI: https://doi.org/10.1515/bgsl.2011.007

Posted at the Zurich Open Repository and Archive, University of Zurich ZORA URL: https://doi.org/10.5167/uzh-154569

Journal Article

Published Version

Originally published at:

Marti, Mirjam (2011). Rolf H. Bremmer, Jr.: An Introduction to Old Frisian. History, Grammar, Reader, Glossary. Beiträge zur Geschichte der deutschen Sprache und Literatur, 133(1):121-124.

DOI: https://doi.org/10.1515/bgsl.2011.007 


\section{BESPRECHUNGEN}

Rolf H. Bremmer, JR.: An Introduction to Old Frisian. History, Grammar, Reader, Glossary, Amsterdam, Philadelphia: John Benjamins 2009, xii, $237 \mathrm{~S}$.

»Das Studium des Afries. hat in den letzten Jahren einen erfreulichen Aufschwung genommen; in weitere Kreise ist es aber immer noch nicht gedrungen, da es an bequemen Hülfsmitteln zur ersten Einführung vollständig fehlt« (Heuser 1903, S. V). Wilhelm Heusers Aussage ist heute - mehr als hundert Jahre später - noch erstaunlich aktuell. Blickt man in die Nachbarphilologien, so stellt man fest, dass für die anderen altgermanischen Sprachen teilweise bereits mehrere Einführungen existieren, die den Einstieg in das Studium ermöglichen sollen. Für das Altfriesische war das Angebot bislang sehr beschränkt. So liegen zwar mit Thomas Markeys >Frisian< (1981) in englischer und Bo Sjölins `Einführung in das Friesische (1969) in deutscher Sprache zwei gute Darstellungen vor, die dem Altfriesischen viel Platz widmen, jedoch liefern beide weder eine Textauswahl noch ein Glossar. In diesem Sinne ebenfalls unvollständig ist Paolo Ramats >Das Friesische (1976), welches zwar Texte bietet, dem jedoch die grammatische Einführung und ein Glossar fehlen. Vergleichbar mit der jetzt vorliegenden >Introduction to Old Frisian < sind von der Konzeption her lediglich Wilhelm Heusers >Altfriesisches Lesebuch (1903), welches sich in erster Linie an Studierende der Altanglistik wendet, sowie Walther Stellers >Abriss der altfriesischen Grammatik (1928); beide stammen jedoch aus der ersten Hälfte des 20. Jahrhunderts. Bremmers Lehrbuch füllt somit vor allem auch im englischsprachigen Raum eine Lücke.

Die Aufgabe des vorliegenden Buches beschreiben Titel und Vorwort folgendermaßen: »[I]t claims to be an introduction to Old Frisian« (S. ix). Es soll den Studierenden des Altfriesischen genügend Informationen liefern, um selbständig Texte lesen und einen Einblick in die Vielfalt der altfriesischen Überlieferung gewinnen zu können (vgl. S. ix). Bei Form und Aufbau hat sich Bremmer nach eigenen Aussagen (S. xi) an Einführungen ins Altenglische orientiert, mit denen er innerhalb seiner Lehrtätigkeit Erfahrungen gesammelt hat. Dies hat sich augenscheinlich bezahlt gemacht. Seine >Introduction to Old Frisian< zeichnet sich durch eine große Benutzerfreundlichkeit aus und ermöglicht damit einen einfachen ersten Einstieg in die Sprache. Sie soll jedoch nicht nur dem Anfänger dienen, sondern auch dem "advanced student who is interested in the earliest period of the Frisian language and/or in comparative Germanic linguistics« (S. x).

Der Aufbau des Buches wird bereits aus dem Untertitel >History, Grammar, Reader, Glossary< ersichtlich. Im Gegensatz zu den bisherigen Einführungsbüchern begnügt sich Bremmer nicht mit der kulturgeschichtlichen und sprachlichen Beschreibung des Altfriesischen, sondern er ergänzt das Lehrbuch um eine Textsammlung, welche in einem Glossar erschlossen wird. Er hat somit die anspruchsvolle Aufgabe übernommen, das Altfriesische in all seiner Komplexität übersichtlich darzustellen. Das erste Kapitel »History. The when, where and what of Old Frisian « (S. 1-19) bietet auf rund 20 Seiten einen ersten Einstieg in Kultur- und Stammesgeschichte, Sprache und Überlieferung, gefolgt von einer knappen forschungsgeschichtlichen Einführung. Praktisch für den Anfänger ist die Auflistung 
der Handschriftensiglen, welche chronologisch eine kurze Übersicht über die Überlieferungssituation bietet.

Dem Charakter einer Einführung entsprechend steht der grammatische Teil (S. 21-108) im Vordergrund; jeweils in Form eines separaten Kapitels wird in die linguistischen Teilbereiche Phonologie, Morphologie, Lexikologie und Syntax eingeführt, wobei ein Schwerpunkt auf der Laut- und Formenlehre liegt. Das Kapitel zur Phonologie beginnt mit einigen einführenden und methodischen Bemerkungen; Bremmer verweist dabei auf die in den sog. nordseegermanischen oder ingwäonischen Sprachen oftmals problematische relative Chronologie der Lautgesetze, die er anhand von Patrick Stiles' Darstellung (Stiles 1995, S. 199f.) präsentiert. Im Zentrum dieses Kapitels stehen "major sound-changes that occurred in the development of West Germanic [...] to Old Frisian « (S. 22). Den phonologischen Ausgangspunkt bildet das westgermanische Vokalsystem; ein wenig unstimmig mag erscheinen, dass auf eine Übersicht des westgermanischen Konsonantensystems verzichtet wird, anschließend jedoch die Darstellung der westgermanischen Konsonantengemination folgt. Die übrigen Lautwandelerscheinungen sind unterteilt in einerseits »Ingvaeonic/North Sea Germanic « und andererseits »Proto-Frisian sound-changes «. Verwirrend ist dabei die Auflistung der 1. Lautverschiebung und Verners Gesetz innerhalb der zweiten Gruppe, also den »Proto-Frisian soundchanges «.

Didaktisch überaus nützlich ist, dass die einzelnen Lautveränderungen immer einheitlich beschrieben werden. So wird jeweils der Lautwandel (»Process«) zuerst ausformuliert; darauf folgen eventuelle Präzisierungen und Beispiele (»Instances«) und eine Einreihung in die relative Chronologie der besprochenen Lautgesetze. Somit ergibt sich eine übersichtliche Einführung in den mannigfaltigen Lautwandel, welcher das Erscheinungsbild des Altfriesischen prägt. Die Seiten 42-51 liefern einen Überblick über das altfriesische Phonemsystem, den Ursprung der einzelnen Laute und einige komplexe Phonem-Graphem-Relationen - bei der relativ komplizierten Phonologie des Altfriesischen für die Studierenden eine wertvolle Übersicht.

Das Kapitel zur Morphologie beginnt mit den Pronomen, gefolgt von den Substantiven, Adjektiven, den Zahlwörtern und Verben. Die Substantivflexion stellt Bremmer im Unterschied zu anderen Darstellungen, die einen synchronen Ansatz verfolgen (vgl. Sjölin 1969, S. 31 f., und Markey 1981, S $119 \mathrm{ff}$.), historisch traditionell dar. So unterscheidet er konsonantische und vokalische Deklination sowie eine dritte Gruppe mit kleineren Flexionsklassen, welche altfriesisch nur noch in Resten bezeugt sind. Wer den Umgang mit historischen Grammatiken der altgermanischen Sprachen - wie beispielsweise mit der >Sammlung kurzer Grammatiken germanischer Dialekte $<$ - gewohnt ist, wird sich rasch zurecht finden und sich einen schnellen Überblick verschaffen können. Die Darstellung der Adjektivflexion ist konzise und übersichtlich. Das Kapitel zu den Verben gliedert sich nach starken und schwachen Verben, Präterito-Präsentien und zuletzt Verben mit besonderer Flexion wie beispielsweise wesa >sein oder $d w \bar{a}>$ tun<. Bei den starken und schwachen Verben wird zuerst die Stammbildung besprochen, die Flexionsparadigmen folgen in einem hinten angefügten Abschnitt »Summary and Paradigms « (S. 84 ff.). Besonders hilfreich ist die Darstellung der Ablautreihen der starken Verben (S. 71), in welcher die Wurzelstruktur und der rekonstruierte germanische Vokalismus der einzelnen Averbostufen jeweils direkt mit dem altfriesischen Lautstand (dargestellt durch die Stammformen eines Beispielverbs) verglichen werden können. Hier haben sich einige kleinere Ungereimtheiten eingeschlichen, welche 
dem fortgeschrittenen Studierenden möglicherweise gar nicht auffallen, dem unerfahrenen jedoch Probleme bereiten können. So findet sich in der Überblicksdarstellung (S. 71) bei der IV. Klasse das Reihenmerkmal germ. * $e+$ Konsonant (nicht wie sonst üblich *e + Resonant) durch afr. stela >stehlen< und nima >nehmen< illustriert, ohne auf die Hebung ${ }^{*} e>i$ in nima einzugehen. Im Paragraphen, in welchem die Klasse näher besprochen wird (S. 74f.), findet sich außerdem kommentarlos als weiteres Beispielverb kuma >kommen< mit schwundstufigem Präsens; indirekt wird zwar gesagt, dass Verben auf einfachen Nasal und Liquid in diese Gruppe fallen - doch wie soll ein Anfänger den plötzlichen Vokalwechsel im Präsens verstehen? Ebenfalls schwierig zu durchschauen ist, dass beispielsweise einmal in der Übersicht nam als die Averbostufe des Prät. Sing. zum Verb nima aufgeführt ist, später jedoch die Flexionsform nom steht. Solche Kleinigkeiten hätten einer Erklärung bedurft. Insgesamt ist die grammatische Darstellung aber sehr präzise und übersichtlich. Der Benutzerfreundlichkeit ist überdies zuträglich, dass weiterführende Informationen, welche vor allem den erfahrenen Studierenden interessieren, durch Kleindruck abgesetzt sind und so dem Benutzer eine Gliederung in Basis- und Detailwissen geboten wird.

Das Kapitel zur Lexikologie (S. 87-96) hat einerseits die Wortbildung und andererseits den Lehnwortschatz des Altfriesischen zum Thema. Es bietet eine Übersicht der Suffixe zur Ableitung von Substantiven (unterteilt in Agens-, Abstraktund Konkretbildungen), Adjektiven und Adverbien, "a fairly complete survey « (S. 91) der altfriesischen Präfixe und einige Anmerkungen zur Komposition. Der Lehnwortschatz wird nach Gebersprache unterteilt und schließt mit ein paar interessanten Beispielen zu Lehnübersetzungen.

Die Syntax (S. 97-108) fällt im Vergleich zur Morphologie relativ knapp aus. Bremmer stellt auch klar, dass eine detaillierte Darstellung den Rahmen eines Einführungsbuches sprengen würde. Er greift deshalb einige syntaktische Phänomene heraus »that might present problems in reading an Old Frisian text« (S. 97). Das gesamte Kapitel bleibt dann auch an der Übersetzungspraxis orientiert. Hilfreich für Studierende ist in diesem Zusammenhang mit Sicherheit die Auflistung der altfriesischen Präpositionen und der Kasus, welche sie regieren können (S. 101).

Die letzten beiden Kapitel runden den grammatischen Teil der Einführung ab: Einerseits wird in die Dialektgliederung des Altfriesischen eingeführt und andererseits wird ein Einblick in die aktuelle Forschungsdebatte gewährt. Kapitel 6 (S. 109-118) liefert dabei eine Übersicht über die linguistischen Merkmale, welche eine Aufteilung in die Mundarten Altost- und Altwestfriesisch begründen; zudem wird die Diskussion aufgegriffen, inwiefern eine wirkliche Dialektgliederung und nicht nur ein chronologisches Phänomen vorliegt. Den Abschluss bilden »two long-standing problems « (S. 119), nämlich die Periodisierung und die Frage nach einer möglichen engeren Verwandtschaft von Altfriesisch und Altenglisch (Kap. 7, S. 119-128).

Der Reader (S. 129-186) umfasst eine Auswahl von Texten, die Bremmer nach Schwierigkeitsgrad ansteigend ordnet (vgl. Einleitung, S. X). Jeder Text wird von einer Einführung und einigen Übersetzungshilfen begleitet. Die einzelnen Lexeme lassen sich problemlos mit Hilfe des Glossars übersetzen. So ist jede Flexionsform, die in den Texten auftaucht, im Glossar mit ihren grammatischen Angaben aufgeschlüsselt (im Rahmen eines einsemestrigen Einführungskurses ins Altfriesische ist mir einzig das Verb snitha >schneiden< untergekommen, welches im Glossar zu fehlen scheint). In Klammern folgt eine etymologische Entsprechung einer 
anderen altgermanischen Sprache, meist des Altenglischen. Die Namen finden sich nicht im Glossar, sondern werden auf S. 220 separat aufgeführt.

Alles in allem liegt mit Bremmers >Introduction to Old Frisian< ein Lehrbuch vor, welches endlich eine lange bestehende Lücke füllt und sowohl den Studierenden wie auch den Dozierenden ein modernes Lehrmittel an die Hand gibt. Nur wenige Kritikpunkte sind zu nennen. Hier und da haben sich etwa kleinere Ungenauigkeiten wie die Rekonstruktion von germ. *seha- ssehen< an Stelle von *seh$w a$ - eingeschlichen (S. 37); manchmal fehlen weitergehende Erklärungen. Weiters verfügt die >Introduction to Old Frisian`zwar über ein Abkürzungsverzeichnis, dieses erschließt jedoch nur die Abkürzungen im Glossar. So bleiben nicht nur sehr gebräuchliche Abkürzungen wie z. B. Gmc für >Germanic ohne Auflösung, sondern auch weniger häufige wie ModWFris für >Modern West Frisian<, IslNFris für >Island North Frisian< oder Wied. für die Wiedingharder Mundart. Nützlich wären auch weitere geographische Karten gewesen. Es finden sich lediglich zwei, eine zur Verteilung der altfriesischen Manuskripte (S. 16), die andere zum Altfriesischen um 1300 (S. 110). Vermisst habe ich eine Übersicht zur Verbreitung der modernen friesischen Dialekte, auf die im ersten Kapitel referiert wird, oder eine Karte, in welcher die Lauwers, der Fluss, welcher allgemein als Grenze zwischen Altost- und Altwestfriesisch gilt, deutlich zu erkennen ist. Dies sind aber kleinere Schwächen im Vergleich dazu, was Bremmers >Introduction to Old Frisian< leistet. Wie bereits erwähnt, habe ich das Buch bereits innerhalb eines Einführungskurses verwendet, wobei es sich im Unterrichtsalltag bewährt hat. Zweifelsfrei liegt ein Lehrbuch vor, welches das Studium des Altfriesischen für Lernende und Lehrende attraktiv macht und die Studierenden für diese wenig beachtete altgermanische Sprache motivieren kann.

\section{LITERATUR}

Heuser, Wilhelm 1903: Altfriesisches Lesebuch. Mit Grammatik und Glossar, Heidelberg (Sammlung germanischer Elementarbücher 3: Lesebücher 1).

Markey, Thomas L. 1981: Frisian, The Hague (Trends in linguistics 13).

Ramat, Paolo 1976: Das Friesische. Eine sprachliche und kulturgeschichtliche Einführung, Innsbruck (Innsbrucker Beiträge zur Sprachwissenschaft 14).

Sjölin, Bo 1969: Einführung in das Friesische, Stuttgart (Sammlung Metzler M 86).

Steller, Walther 1928: Abriss der altfriesischen Grammatik, Halle.

Stiles, Patrick V. 1995: Remarks on the >Anglo-Frisian< Thesis, in: Volkert F. Faltings [u.a.] (Hgg.): Friesische Studien II. Beiträge des Föhrer Symposiums zur Friesischen Philologie vom 7.-8. April 1994, Odense (NOWELE. Suppl. 12), S. 177-220.

lic. phil. Mirjam Marti, Deutsches Seminar der Universität Zürich, Schönberggasse 9, CH-8001 Zürich; m.marti@ds.uzh.ch. 